\title{
TTR
}

Traduction, terminologie, rédaction

\section{Robert Carvais, Valérie Nègre, Jean-Sébastien Cluzel et Juliette Hernu-Bélaud, dir. Traduire l'architecture. Texte et image : un passage vers la création? Paris, Picard, 2015, 296 p.}

\section{Yves Chevrel}

Volume 29, numéro 1, 1er semestre 2016

URI : https://id.erudit.org/iderudit/1050715ar

DOI : https://doi.org/10.7202/1050715ar

Aller au sommaire du numéro

\section{Éditeur(s)}

Association canadienne de traductologie

ISSN

0835-8443 (imprimé)

1708-2188 (numérique)

Découvrir la revue

Citer ce compte rendu

Chevrel, Y. (2016). Compte rendu de [Robert Carvais, Valérie Nègre, Jean-Sébastien Cluzel et Juliette Hernu-Bélaud, dir. Traduire l'architecture. Texte et image : un passage vers la création ? Paris, Picard, 2015, 296 p.] TTR, 29(1), 245-249. https://doi.org/10.7202/1050715ar d'utilisation que vous pouvez consulter en ligne.

https://apropos.erudit.org/fr/usagers/politique-dutilisation/ 


\section{COMPTES RENDUS}

Robert Carvais, Valérie Nègre, Jean-Sébastien Cluzel et Juliette Hernu-Bélaud, dir. Traduire l'architecture. Texte et image : un passage vers la création? Paris, Picard, 2015, 296 p.

L'architecture ressortit à l'art et à la science et concerne la vie sociale. Sur ces bases, l'ouvrage analysé ici met en évidence les spécificités des traductions d'ouvrages d'architecture. Issu d'une entreprise engagée en 2009, appuyé sur une série de journées d'étude, il réunit 21 contributions (17 en français et 4 en anglais) réparties en deux grandes parties équilibrées : "Traduire, d'une langue à l'autre» et "La traduction comme pratique créative». Celles-ci sont encadrées par des «Remarques sur quelques particularités de la traduction en architecture» (Nègre, p. 9-15) et une Postface intitulée "L'architecture 'traduite', entre fidélité et innovation?» (Carvais, p. 271-287). Un Index des noms et des institutions (p. 289-296) clôt le volume, qui inclut de nombreuses reproductions de planches.

Cette structure n'implique toutefois pas une distinction radicale entre deux types de traductions. Très bien informée sur les débats théoriques actuels en traductologie et leurs enjeux, la Postface explicite les apports des différents contributeurs. Robert Carvais y propose une typologie complexe de la traduction architecturale: à côté de la traduction altérante, déformant sciemment l'ouvrage pour des raisons parfois difficiles à établir, il distingue : la traduction savante, attachée à transmettre des débats théoriques; la traduction technique, utile pour l'action (construire, mais aussi bâtir une nomenclature juridique); la traduction politique, au service de missions d'État; la traduction primaire ou littérale, visant à faire comprendre un ouvrage consacré à un sujet précis, adapté pour des motifs culturels. Ces différents types de traduction peuvent évidemment se recouper ou se superposer; ils impliquent de plus les questions liées à la «traduction visuelle» des illustrations.

Ces questions traversent la grande majorité des contributions qui constituent l'ouvrage. L'article «The Tomb of Porsenna» de Fabio Colonnese part de la description, due à Pline l'Ancien, $\mathrm{du}$ Labyrinthus Italicus, dont l'existence n'est pas avérée, pour évoquer diverses «graphical translations» (p. 161) (qui supposent une possible altération des manuscrits: petasus ou pegasus?). 
À l'inverse, explique Michaël Decrossas dans son article «Les œuvres d'architecture d'A. Le Pautre», les dessins d'Antoine Le Pautre, publiés en planches de 1652 à 1658 , sont réédités après sa mort, vers 1683, avec des «discours» (p. 182) du jeune AugustinCharles d'Aviler, qui sous-entend leur aspect utopique.

Le rapport texte-image est évidemment au cœur de tous les articles qui étudient des traductions précises; les langues concernées, langues sources et/ou langues cibles, sont nombreuses : latin, italien, français, anglais, allemand, espagnol, roumain, néerlandais, suédois, japonais. Le latin est la langue du premier traité d'architecture conservé en Europe, le De architectura de Vitruve: vers 1452, Leon Battista Alberti s'efforce de créer un latin technique notamment dans le De re aedificatoria, prévu pour remplacer l'ouvrage de Vitruve, critiqué pour son vocabulaire trop hellénisé, ce qui conduit Pierre Caye, dans son article «L. B. Alberti et la question de la latinité technique», à mettre en cause l'opinion reçue selon laquelle les langues vernaculaires jouent un rôle primordial à la Renaissance. En 1708 encore, Henry Aldrich publie ses Elementa Architecturae pour servir d'exemple aux débutants; il y exprime sa révérence envers Palladio (autre grande référence), mais en s'écartant fortement de ses dessins, comme le montre Eleonora Pistis dans son article «'Tironibus pro exemplo': Henry Aldrich's Elementa Architecturae and architectural education at Oxford». Mais, très vite, la circulation européenne des ouvrages en langues vernaculaires pose de nouveaux problèmes.

Jean Rondelet (1743-1829) fait partie des architectes reconnus, notamment pour son Traité théorique et pratique de l'art de bâtir (1807-1817). Très souvent réédité, l'ouvrage est traduit en italien (1831-1835) et en allemand (1833-1836). Fondées toutes deux sur la $5^{\mathrm{e}}$ édition (revue, 1827-1832), ces deux entreprises sont étudiées, l'une par Valérie Nègre («Italianiser Rondelet. De l'Art de bâtir à l'Arte di edificare»), l'autre par Torsten Meyer («Reading Footnotes and Comments. Some Remarks on the German Translation of J. Rondelet's Traité»). La traduction italienne, due à Basilio Soresina, paraît dans une «Biblioteca scelta dell'Ingegnere Civile» (16 traités, dont 8 traduits du français); le traducteur joint des notes personnelles, identifiées comme telles, et ajoute 23 planches aux quelque 200 de Rondelet; tout en suivant les perspectives scientifiques de celui-ci, il utilise d'autres auteurs, notamment quand il s'agit des routes et des ponts : l'équipement 
du territoire est une des finalités de la collection. La Theoretischpraktische Anleitung zur Kunst zu bauen est l'œuvre de Carl Heinrich Distelbarth et de Johann Friedrich Christian Hess, architectes qui ont vécu à Paris; les traducteurs recourent à des notes infrapaginales, pour corriger des erreurs (comme l'inversion d'une image rendant le texte incompréhensible), discuter des interprétations des textes cités par Rondelet, avancer des compléments dus aux progrès de l'art de bâtir, expliciter certaines traductions dans des domaines peu connus des lecteurs allemands, proposer d'autres exemples (constructions en bois en Allemagne). Sans chercher à altérer le texte original, ils se comportent en lecteurs critiques.

Autre architecte français traduit: Eugène-Emmanuel Viollet-le-Duc (1814-1879). Plusieurs de ses ouvrages ont été traduits quasi simultanément en anglais. Si, en Grande-Bretagne, Entretiens sur l'architecture (1863) est rendu par Lectures on Architecture, aux États-Unis, il l'est par Discourses on Architecture; Histoire d'une maison, titré en Grande-Bretagne How to Build a House. An Architectural Novelette, devient outre-Atlantique The Story of a House. Dans son article "Traduire Viollet-le-Duc en anglais : modalités et enjeux», Isabelle Gournay étudie ce cas qui montre une réelle proximité entre l'Angleterre et l'œuvre de Viollet-le-Duc, tandis que les Américains restent, aujourd'hui encore, un peu en retrait.

Trois articles mettent spécialement en lumière les problèmes liés aux traductions allemand-français, dans les deux sens. Martin Pozsgai, dans «La traduction du Cours d'architecture de d'Aviler par L. C. Sturm», montre que le traducteur allemand Leonhard Christoph Sturm estime que la connaissance de la matière est plus importante que celle de langue, et qu'il tend à germaniser l'ouvrage de d'Aviler. Pour sa part, Véronique Samuel-Gohin étudie "Terminologie et concepts d'architecture entre l'allemand et le français» à partir d'adaptations française du traité de Christian Wolff Anfangsgründe aller mathematischen Wissenschaften et allemande de Jean-Nicolas-Louis Durand Précis des leçons d'architecture [...] : elle y montre comment le dessin «constitue un moyen privilégié de traduire les concepts architecturaux» (p. 70). Christoph Schnoor étudie «Le Raum dans La construction des villes de Le Corbusier» : ce concept allemand généralement traduit par espace renvoie plutôt, en architecture, à une impression de volume, que corporalité pourrait rendre. 
Deux contributions abordent une rencontre compliquée entre Europe et Asie : «Traduire l'architecture japonaise au XIX ${ }^{\mathrm{e}}$ siècle», de Jean-Sébastien Cluzel, et «L'édition japonaise du Vignole à la fin du XIX ${ }^{\mathrm{e}}$ siècle», de Nishida Masatsugu.

D'autres pratiques traductives sont analysées : comment, en Suède, on élabore une sorte de "manuel national de construction" (p. 225) à partir de modèles étrangers (Linnéa Rollenhagen Tilly, «Le premier recueil d'architecture illustré suédois. C. Wijnblad»); comment, aux Pays-Bas, on s'inspire de manuels français (Petra Brouwer, «Appropriating New Standards. The Influence of French Books on the First Architectural Manuals in the $19^{\text {th }}$ Century»). On découvre aussi une traduction « mosaïque » de textes (p. 213) qui peut entraîner des retraductions (Susanna Pasquali, «F. Milizia, le traducteur traduit»), voire des traductions «impossibles», comme le montrent Olga Medvedkova («Scamozzi en français : histoire d'un échec»), et Juliette Hernu-Bélaud, qui parle de «traductions éditoriales» («L'Architecture pratique de P. Bullet: la traduction impossible et ses variantes intra-linguistiques»).

Sous-jacents à toutes ces perspectives, les problèmes lexicaux font l'objet de considérations portant sur «l'expression 'architecture romane' et ses traductions» que Leu Huang étudie au XIX ${ }^{\mathrm{e}}$ siècle, ou sur «la terminologie architecturale roumaine au $\mathrm{XIX}^{\mathrm{e}}$ siècle» (Muguraş et Lucian Constantinescu). Ils incluent aussi - ce n'est pas la moindre difficulté - le vocabulaire du droit. Dans ce domaine, l'article «Sive gallice» de Philippe Bernardi, examinant des contrats notariaux du Comtat Venaissin et du Comté de Provence, montre comment des notaires formés en droit mais non en architecture essaient de rendre clairs les actes qu'ils rédigent, en ajoutant des termes de la langue vulgaire. De son côté, Robert Carvais, au travers de rééditions du cours d'Antoine Desgodets édité en 1748, pose la question : "Comment traduire les Loix des Bâtimens après l'avènement du Code civil?" et conclut à une possible distinction entre deux types de traducteurs: «juriste sourcier» et «architecte cibliste» (p. 207).

Ce panorama des livres architecturaux traduits est riche et il ouvre sur de nombreuses pistes de recherche. L'architecture, vue au prisme de la traduction, est bien un art et une science : en cela les pratiques s'inscrivent parfaitement dans l'histoire des traductions scientifiques (voir les chapitres des volumes correspondants dans l'Histoire des traductions en langue française, 2012-2015). Mais 
le lien nécessairement très fort entre textes et images constitue un iconotexte, dont l'exploration est assurément un domaine de recherche d'avenir du XXI' siècle.

\section{Références}

Chevrel, Yves, Lieven D'hulst et Christine Lombez, dir. (2012). Histoire des traductions en langue française. XIX ${ }^{e}$ siècle. Lagrasse, Verdier.

Chevrel, Yves, Annie Cointre et Yen-Maï Tran-Gervat, dir. (2014). Histoire des traductions en langue française. XVII et $X V I I I^{e}$ siècles. Lagrasse, Verdier.

Duché, Véronique, dir. (2015). Histoire des traductions en langue française. $X V^{e}$ et $X V T^{e}$ siècles, Lagrasse, Verdier.

Yves Chevrel

Université Paris-SorbonNe

\section{Jean Delisle et Marco A. Fiola. La traduction raisonnée. Manuel} d'initiation à la traduction professionnelle de l'anglais vers le français. $3^{\mathrm{e}}$ édition. Ottawa, Presses de l'Université d'Ottawa, collection «Pédagogie de la traduction", 2013, 716 p.

Depuis la parution de la première édition en 1993, cet ouvrage a fait école (dans son compte rendu, Michel Ballard [1994, p. 487] y discerne déjà un certain classicisme) et façonné l'enseignement de la traduction professionnelle au Canada et ailleurs dans le monde. À preuve : la genèse de cet ouvrage, soit l'Analyse du discours comme méthode de traduction publiée en 1980 par Jean Delisle, a été traduite en anglais, en chinois, en espagnol, en persan et en turc, et tout indique que l'ouvrage a aussi servi de modèle dans la conception d'un autre manuel sur la traduction de l'espagnol à l'anglais publié par Allison Beeby-Lonsdale (1996) dans la collection «Pédagogie de la traduction», et qui reprend la formule des objectifs d'apprentissage fondée sur la taxonomie de Bloom en pédagogie.

La troisième édition parue à la fin de 2013 mise sur ce patrimoine et vise à le mettre en valeur tout en proposant des améliorations - déplacement de quelques objectifs ainsi que du glossaire, et renouvellement du contenu de la rubrique «Suggestion(s) de lecture» proposée dans chacun des objectifs pédagogiques - et plusieurs nouveautés. Mentionnons d'abord l'apport d'un coauteur, Marco A. Fiola, professeur de traduction 